\title{
SAX-PAC (Scalable And eXpressive PAcket Classification)
}

\author{
Kirill Kogan \\ Purdue University and \\ NetSysAlgo \\ kirill.kogan@gmail.com
}

\author{
Sergey Nikolenko \\ Steklov Mathematical Institute \\ at St. Petersburg, \\ National Research University \\ Higher School of Economics \\ sergey@logic.pdmi.ras.ru
}

\author{
Ori Rottenstreich \\ Mellanox, Israel \\ orir@mellanox.com
}

\author{
William Culhane \\ Purdue University \\ wculhane@purdue.edu
}

\author{
Patrick Eugster \\ Purdue University and \\ Technical University of \\ Darmstadt \\ peugster@cs.purdue.edu
}

\begin{abstract}
Efficient packet classification is a core concern for network services. Traditional multi-field classification approaches, in both software and ternary content-addressable memory (TCAMs), entail tradeoffs between (memory) space and (lookup) time. TCAMs cannot efficiently represent range rules, a common class of classification rules confining values of packet fields to given ranges. The exponential space growth of TCAM entries relative to the number of fields is exacerbated when multiple fields contain ranges. In this work, we present a novel approach which identifies properties of many classifiers which can be implemented in linear space and with worst-case guaranteed logarithmic time and allows the addition of more fields including range constraints without impacting space and time complexities. On real-life classifiers from Cisco Systems and additional classifiers from ClassBench [7] (with real parameters), $90-95 \%$ of rules are thus handled, and the other 5$10 \%$ of rules can be stored in TCAM to be processed in parallel.
\end{abstract}

\section{Categories and Subject Descriptors}

C.2.6 [Computer-Communication Networks]: InternetworkingRouters

\section{Keywords}

packet classification; TCAM

\section{INTRODUCTION}

Packet classification is a core functionality for implementing popular commodity services including Quality of Service (QoS) and access control. Recently packet classification has become even more prominent with the adoption of OpenFlow [25], which expresses features through hierarchical tuple matching with set actions. Multi-field classification, i.e. classification based on more

Permission to make digital or hard copies of all or part of this work for personal or classroom use is granted without fee provided that copies are not made or distributed for profit or commercial advantage and that copies bear this notice and the full citation on the first page. Copyrights for components of this work owned by others than ACM must be honored. Abstracting with credit is permitted. To copy otherwise, or republish, to post on servers or to redistribute to lists, requires prior specific permission and/or a fee. Request permissions from permissions@ acm.org.

SIGCOMM'14, August 17-22, 2014, Chicago, IL, USA.

Copyright 2014 ACM 978-1-4503-2836-4/14/08 ...\$15.00.

http://dx.doi.org/10.1145/2619239.2626294 . than one field, has become very common. If a classification rule sets exact values for all fields, the rule can be represented by concatenating all its fields. Such classifiers can be implemented in content-addressable memory (CAM) or by a simple hash function in space linear in the number of fields. The problem becomes harder if a field is represented by a prefix (constraint on most significant bits) or a range (confining values inside an interval) since simple concatenation stops working.

Many sophisticated software-based approaches have been proposed (cf. comprehensive survey [40]). Complexity bounds derived from computational geometry imply that a software-based packet classifier with $N$ rules and $k \geq 2$ fields uses either $O\left(N^{k}\right)$ space and $O(\log N)$ time or $O(N)$ space and $O\left(\log ^{k-1} N\right)$ time [26]. Thus, software-based approaches are either too slow or too memory-intensive even with few prefix- or range-based fields.

Ternary content-addressable memory (TCAM) was introduced to overcome performance problems for prefix matching [24]. TCAM is a fast class of memory for matching packet headers against a set of stored entries represented by tuples of VALUE and MASK words (the latter hides "don't care" bits). Currently, TCAM is the de facto standard for classifier implementation. Unlike softwarebased solutions, TCAM can efficiently represent multi-field classification with prefixes, but suffers an exponential memory blowup from range expansion; each range-based field in a classification rule introduces an additional multiplicative factor. Thus in practice the number of range fields is severely limited.

Defining more sophisticated services requires additional expressiveness on existing classification fields or new ones, and many classifications are naturally represented by ranges. More efficient implementations for classifiers on ranges of IP or MAC addresses, dates, packet lengths, etc. are already highly desired [8]. The stateof-the-art packet classification mechanisms are limited and mostly consider five-tuples with at most two fields which include ranges. This greatly restricts emerging classification possibilities.

In this paper we consider a hybrid software- and TCAM-based approach, which hinges on the order-independence of classifiers with respect to the rules they encompass. We identify this property as essential for simple and efficient implementation of classifiers. We show that if a classifier is order-independent then the addition of new classification fields - even those represented by ranges does not increase the complexity of lookup time or memory space required. We use this to build efficient classification schemes. We also consider the general case with order-dependence, leading to a hybrid approach implementing order-independent rules in software 
using linear memory and with logarithmic worst-case guaranteed lookup time, and the rest of the rules in TCAM. More precisely, our contributions are the following: (1) we identify classifier properties that guarantee zero cost for additional fields represented by ranges or prefixes transparently to the implementation scheme; (2) we propose a technique to reduce the number of classification fields represented by prefixes or ranges, yielding semantically equivalent classification results; (3) we present a technique to reduce a multi-field classification problem to a classification problem on at most two fields with guaranteed worst-case performance and linear memory which is more sophisticated than previous reducation attempts which do not provide acceptable worst-case guarantees when classifiers start with more than two fields [35, 36]; (4) we demonstrate the viability of our approach through simulations on 12 classifiers from Classbench generated with real parameters [7], each with $\approx 50 \mathrm{~K}$ rules on 6 fields and on 5 real-life classifiers from Cisco Systems; we show that up to $90-95 \%$ of the rules can be implemented in software in linear space and with worst-case guaranteed logarithmic time, leaving only $5-10 \%$ to be implemented in TCAM.

The rest of this paper is organized as follows. Section 2 details the model underlying our work. Section 3 explains the relevance of order-independence, and Section 4 introduces the main computational problems underlying our approach. Section 5 deals with the Boolean representation of classifiers, comparing it with our approach, whereas Section 6 presents heuristics to efficiently find suboptimal solutions for the computational problems and Section 7 proposes new standard representations of classifiers. Section 8 presents simulation results. Section 9 discusses related prior art and Section 10 concludes the paper.

\section{MODEL DESCRIPTION}

Packets are matched by their headers according to classification rules stored in a classification database. A packet header contains $k$ fields; a field $i, 1 \leq i \leq k$, is a string of $W_{i}$ bits. A classifier $\mathcal{K}$ is an ordered set of $\bar{N}$ rules, denoted $R_{1} \ldots R_{N}$. A rule $R_{j}$ is an ordered set of $k$ fields and an associated action $A_{j}$; the $i$-th field $F_{i}$ is represented by a range of values on $W_{i}$ bits, i.e., each rule contains $k$ ranges $R=\left(I_{1}, \ldots, I_{k}\right), I_{i}=\left[l_{i}, u_{i}\right]$. We assume the last rule of each $\mathcal{K}$ is a "catch-all" rule $R_{N}=(*, \ldots, *)$ with $A_{N}=$ TRANSMIT to transmit matched packets without changes. We denote by $\mathcal{K}^{-F}$ the classifier that results from $\mathcal{K}$ by removing the set of fields $F$ from each of its rules; we also usually denote by $\mathcal{K}^{+F}$ a classifier that results from $\mathcal{K}$ by extending its rules with the set of fields $F$ (with values defined separately for every rule), and simplify the notation to $\mathcal{K}^{-|F|}$ and $\mathcal{K}^{+|F|}$ where the fields themselves are clear from context.

Rules are applied sequentially, i.e., the set of rules has non-cyclic ordering $\prec$, and if a header matches both $R_{x}$ and $R_{y}$ for $x \prec y$, rule $R_{x}$ takes precedence. Classifiers are semantically equivalent if they match the same rule for every packet.

A classifier $\mathcal{K}$ is called order-independent if for any classifier $\mathcal{K}^{\prime}$ with the same rules in a different order (except the last "catch-all" rule) and for any packet header $p, p$ is matched by the same rule in both $\mathcal{K}$ and $\mathcal{K}^{\prime}$. This condition for order-independence of a classifier is satisfied exactly when each pair of its rules do not intersect, i.e. for each pair of rules there is at least one field in which the two corresponding ranges (or prefixes) are disjoint. Otherwise, a classifier is called order-dependent. For instance, the classifier $\mathcal{K}$ that contains two rules that are based on two fields $R_{1}=([1,3],[4,5])$ and $R_{2}=([5,6],[4,5])$ is order-independent but $\mathcal{K}^{\prime}$ that contains two rules $R_{3}=([1,3],[4,5])$ and $R_{4}=([2,4],[4,5])$ is orderdependent. This is because $R_{1}, R_{2}$ do not intersect since the ranges
- - - "Regular" TCAM space, SRGE encoding "Regular" TCAM space, binary encoding - - - TCAM space by Theorem 1, SRGE encoding — TCAM space by Theorem 1, binary encoding

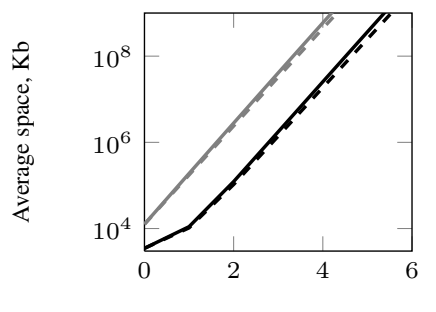

16-bit range fields, ClassBench

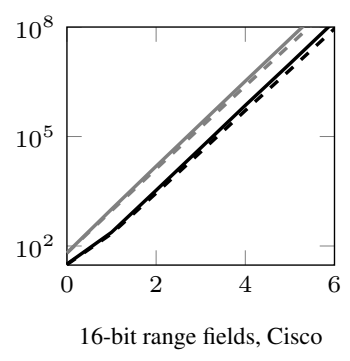

Figure 1: TCAM space required for ClassBench and CISCO classifiers as a function of the number of additional synthetic 16-bit range fields.

in their first field are disjoint while $R_{3}, R_{4}$ do intersect. For instance, the input $(3,4)$ matches both rules of $\mathcal{K}^{\prime} . \mathcal{K}(S)$ denotes a classifier that uses only a subset $S$ of fields in classification.

\section{IMPACT OF ORDER-INDEPENDENCE}

Order-independence of classifiers, or parts thereof, is a prevalent and powerful property for efficient classifier representation and evaluation. Classifiers using longest-prefix matches on a single field are at least order-independent among all prefixes of the same length; forwarding entries in OpenFlow [25] likely preserve orderindependence for simplicity of management; many classifiers representing services and implement "best-match" priority schemes are order-independent. Order-dependent classifiers representing service-level-agreements (SLAs) are often unintended byproducts of uniting several services in a single classifier. In our experience of over a decade of deployments, many classifiers representing services such as QoS or ACLs are order-independent in practice. Increasing the number of rules of a classifier decreases the chance that classifier as a whole will stay order-independent, but the size of its maximal order-independent part increases. Adding fields to each rule increases the chances of order-independence.

To study the impact of order-independence, we analyze classifiers from ClassBench generated with real parameters [7] and reallife classifiers provided by Cisco Systems. The data in Table 1 indicate new methods for efficient representation of order-independent parts of classifiers can greatly reduce requirements for classification engines. If a classifier is not wholly order-dependent, we can lookup its order-independent and order-dependent parts separately and return the highest-priority match. An order-independent match preempts the need to match the order-dependent part. Alternatively, we can choose rules for the order-independent part which have higher priorities than any rule in the other part.

EXAMPLE 1. Consider an order-independent classifier $\mathcal{K}=$ $\left(R_{1}, R_{2}, R_{3}\right)$ with two fields of 5 bits each and rules

$$
\begin{aligned}
& R_{1}=([1,3],[4,31]), \\
& R_{2}=([4,4],[2,30]), \\
& R_{3}=([7,9],[5,21]) .
\end{aligned}
$$

Let $\mathcal{K}^{+1}$ be a classifier that results from $\mathcal{K}$ by adding one new field of 5 bits, leading to the rules

$$
\begin{aligned}
& R_{1}^{+1}=([1,3],[4,31],[1,28]), \\
& R_{2}^{+1}=([4,4],[2,30],[4,27]), \\
& R_{3}^{+1}=([7,9],[5,21],[3,18]) .
\end{aligned}
$$




\begin{tabular}{|c|c|c|c|c|c|c|c|c|c|c|c|c|c|c|}
\hline & \multicolumn{2}{|c|}{ Rules } & \multicolumn{6}{|c|}{ Original classifier } & \multicolumn{6}{|c|}{ Classifier expanded by two 16-bit ranges } \\
\hline & \multirow{3}{*}{ Total } & \multirow{3}{*}{$\begin{array}{l}\text { order-ind., } \\
\text { all fields }\end{array}$} & \multicolumn{3}{|c|}{ Original $\mathcal{K}$} & \multicolumn{3}{|c|}{$\begin{array}{l}\text { By Theorem } 2 \\
\text { Order-ind. }\end{array}$} & \multicolumn{3}{|c|}{ Original $\mathcal{K}^{+2}$} & \multirow{2}{*}{\multicolumn{3}{|c|}{$\begin{array}{l}\text { By Theorem } 1 \\
\text { Order-ind. } \\
\text { width, } \quad \text { Space, } \mathrm{Kb}\end{array}$}} \\
\hline & & & \multirow{2}{*}{$\begin{array}{l}\text { Width, } \\
\text { bits }\end{array}$} & \multicolumn{2}{|c|}{ Space, Kb } & \multirow{2}{*}{$\begin{array}{l}\text { width, } \\
\text { bits }\end{array}$} & \multicolumn{2}{|c|}{ Space, $\mathrm{Kb}$} & \multirow{2}{*}{$\begin{array}{l}\text { Width, } \\
\text { bits }\end{array}$} & \multicolumn{2}{|c|}{ Space, $\mathrm{Kb}$} & & & \\
\hline & & & & Binary & SRGE & & Binary & SRGE & & Binary & SRGE & $\begin{array}{l}\text { w1dth, } \\
\text { bits }\end{array}$ & Binary & SRGE \\
\hline acl1 & 49870 & 49779 & 120 & 7922 & 7655 & 31 & 1517 & 1517 & 152 & 1752769 & 1462501 & 31 & 3857 & 3509 \\
\hline acl2 & 47276 & 44178 & 120 & 11289 & 11289 & 82 & 4189 & 4189 & 152 & 2499525 & 2159699 & 82 & 145925 & 126505 \\
\hline $\mathrm{acl} 3$ & 49859 & 47674 & 120 & 10771 & 10571 & 91 & 5018 & 5008 & 152 & 2391264 & 2027239 & 91 & 177771 & 152514 \\
\hline acl4 & 49556 & 46670 & 120 & 10079 & 9904 & 97 & 5379 & 5370 & 152 & 2234839 & 1895078 & 97 & 216994 & 186701 \\
\hline acl5 & 40362 & 38962 & 120 & 6121 & 6121 & 63 & 2950 & 2950 & 152 & 1359256 & 1172403 & 63 & 127818 & 110663 \\
\hline fw1 & 47778 & 43675 & 120 & 19454 & 19438 & 72 & 3926 & 3911 & 152 & 4303234 & 3720573 & 72 & 194313 & 164893 \\
\hline fw2 & 48885 & 48826 & 120 & 10866 & 10866 & 52 & 2498 & 2498 & 152 & 2399603 & 2071400 & 52 & 7382 & 6695 \\
\hline fw3 & 46038 & 41615 & 120 & 15090 & 15073 & 84 & 4161 & 41 & 152 & 3337763 & 2873120 & 84 & 170688 & 144596 \\
\hline fw4 & 45340 & 42857 & 120 & 33500 & 33368 & 76 & 4025 & 4008 & 152 & 7438741 & 6390798 & 76 & 195130 & 165332 \\
\hline fw5 & 45723 & 39962 & 120 & 12478 & 12445 & 76 & 3759 & 3745 & 152 & 2745105 & 2366939 & 76 & 180420 & 152652 \\
\hline $\mathrm{c} 1$ & 49840 & 48294 & 120 & 8041 & 7924 & 50 & 2580 & 2579 & 152 & 1789100 & 1521153 & 50 & 52378 & 45391 \\
\hline ipc2 & 50000 & 50000 & 120 & 5859 & 5859 & 36 & 1757 & 1757 & 152 & 1301839 & 1123612 & 36 & 1757 & 1757 \\
\hline ciscol & 584 & 538 & 120 & 78 & 78 & 52 & 34 & 34 & 152 & 17523 & 15088 & 52 & 1650 & 1441 \\
\hline $\operatorname{cisco} 2$ & 269 & 249 & 120 & 68 & 68 & 21 & 7 & 7 & 152 & 15662 & 13510 & 21 & 477 & 415 \\
\hline $\operatorname{cisco} 3$ & 95 & 92 & 120 & 11 & 11 & 30 & 3 & 3 & 152 & 2505 & 2137 & 30 & 76 & 71 \\
\hline $\operatorname{cisco} 4$ & 364 & 329 & 120 & 79 & 79 & 38 & 18 & 18 & 152 & 17827 & 15385 & 38 & 1484 & 1237 \\
\hline $\operatorname{cisco} 5$ & 148 & 120 & 120 & 19 & 19 & 17 & 5 & 5 & 152 & 4303 & 3717 & 17 & 695 & 590 \\
\hline
\end{tabular}

Table 1: Space comparison. Left to right: number of rules (total and in a maximal order-independent set); a comparison of TCAM space requirements under two encodings (binary [36] and SRGE [3]) for ClassBench classifiers with standard representation and with a representation reduced by Theorem 2; a similar TCAM space comparison for the same classifiers extended with two additional random synthetic 16-bit range fields, with standard representation and with a representation reduced by Theorem 1 .
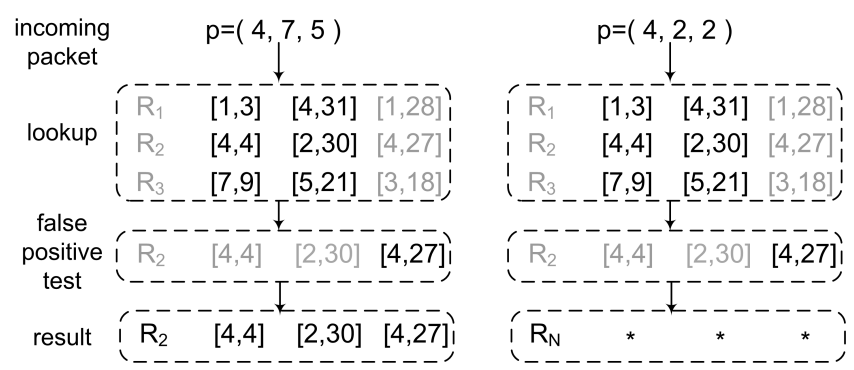

Figure 2: Visualizing the lookup procedure on rules with a subset of fields in Example 1. Fields checked in a certain step are in black; those irrelevant for the step, in grey. Packet $(4,2,2)$ matches $R_{2}$ but fails the false positive check on the added field of $R_{2}^{+1}$, so the classifier returns the catch-all rule.

Since $\mathcal{K}$ is order-independent, the new fields can be skipped during (the main TCAM-based) classification and a single matched rule verified to avoid false positive matches (see Figure 2). The binary encoding [36] of $\mathcal{K}^{+1}$ requires $42+28+50=120$ TCAM entries and the SRGE encoding [3] requires $24+8+32=64$ entries. On the other hand, the binary and SRGE encodings of $\mathcal{K}$ require only $6+7+10=23$ and $6+4+8=18$ TCAM entries respectively.

THEOREM 1 (FIELDS EXPANSION). Let $\mathcal{K}^{+m}$ be a classifier that results from an order-independent classifier $\mathcal{K}$ by adding $m$ new fields of any width, i.e., a rule $R=\left(I_{1}, \ldots, I_{k}\right)$ in $\mathcal{K}$ is replaced with $R^{+m}=\left(I_{1}, \ldots, I_{k}, \ldots, I_{k+m}\right)$ in $\mathcal{K}^{+m}$. Then $\mathcal{K}$ with a false-positive check of a single matched rule is a semantically equivalent representation of $\mathcal{K}^{+m}$.

PROOF. Adding new fields to an order-independent classifier preserves its order-independence, so $\mathcal{K}^{+m}$ is order-independent. If a header $H$ does not match any rule of $\mathcal{K}, H$ cannot match $\mathcal{K}^{+m}$ (we only add constraints). If a header $H$ matches $R_{j} \in \mathcal{K}$, either $H$ matches $R_{j}^{+m} \in \mathcal{K}^{+m}$ or $H$ does not match any rule of $\mathcal{K}^{+m}$. If $R_{j} \in \mathcal{K}$ is matched we check $m$ additional fields to avoid a false-positive match.

By Theorem 1, introducing additional fields based on prefixes or ranges to an order-dependent classifier affects only the encod- ing size of its order-dependent part. The space and lookup time complexity of classification in the order-independent do not increase since new fields in the order-independent part can be ignored without affecting the classification outcome; an additional check of at most one matched rule is required to avoid a false positive match. Previously new fields based on prefixes or ranges significantly increased the complexity of software-based solutions (recall $O\left(\log ^{k-1} N\right)$ lookup time in linear memory). Likewise, in TCAMbased solutions each range is converted to prefixes before configuration, and any new field based on ranges adds an additional multiplicative factor for the required TCAM space. This contributes to the fact that 5-tuple classifiers with only source and destination port fields represented by ranges are the current industry standard. Support for additional fields amenable to range rules would greatly improve classification expressiveness (e.g., ranges on dates, packet length, etc.).

The effect of Theorem 1 is shown in Table 1. We add two new ranges on 16 bits each to benchmark classifiers, garnering significant space savings. We compare memory requirements which result from Theorem 1 with those of two well-known range encoding schemes: binary [36] and SRGE [3]. In addition, Figure 1 demonstrates how average required space depends on the number of range-based fields; for our approach, the exponential growth is significantly deterred. Whereas Theorem 1 shows an equivalent representation of $\mathcal{K}^{+m}$ when new fields are added, Theorem 2 states we can ignore a subset of fields if the remaining fields maintain the order-independence of the classifier. Table 1 shows the effect of Theorem 2: for most benchmarks, significant space savings (by a factor of 2 to 5 ) caused by order-independence result immediately, on the classifiers themselves rather than their extended versions. The effect is independent of range encoding schemes.

EXAMPLE 2. Consider a classifier $\mathcal{K}=\left(R_{1}, R_{2}, R_{3}\right)$ with three fields of 5 bits each and rules

$$
\begin{aligned}
& R_{1}=([1,3],[4,31],[1,28]), \\
& R_{2}=([4,4],[2,30],[4,27]), \\
& R_{3}=([7,9],[5,21],[3,18]) .
\end{aligned}
$$

In this case the first field is necessary but also sufficient to guarantee order independence, so we have that $\mathcal{K}^{-\{2,3\}}=$ 


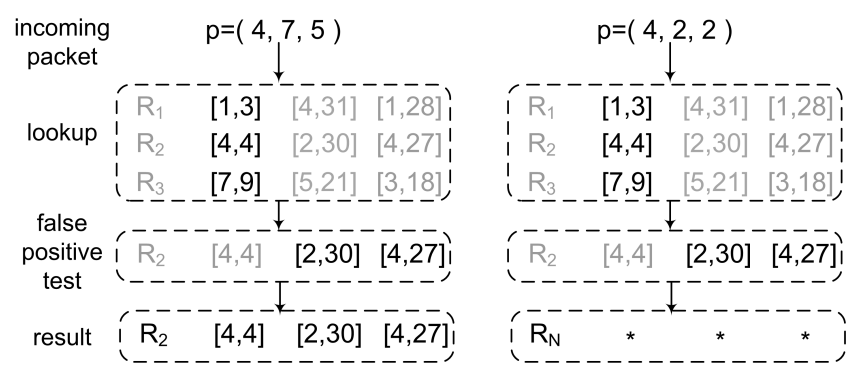

Figure 3: Visualizing the lookup procedure on rules with a subset of fields in Example 2. Fields to be checked in a step are shown in black; irrelevant for the step, in grey. Note that packet $(4,2,2)$ matches $R_{2}^{-\{2,3\}}$ but fails the false positive check, so the classifier returns the catch-all rule.

$\left\{R_{1}^{-\{2,3\}}, R_{2}^{-\{2,3\}}, R_{3}^{-\{2,3\}}\right\}=\{([1,3]),([4,4]),([7,9])\}$ is order-independent (see Figure 3 ). That the binary encoding of $\mathcal{K}$ requires $42+28+50=120$ TCAM entries and the SRGE encoding of $\mathcal{K}$ requires $24+8+32=64$ TCAM entries. On the other hand, the binary encoding for $\mathcal{K}^{-\{2,3\}}$ requires $2+1+1=4$ TCAM entries and the SRGE encoding requires only $2+1+2=5$ TCAM entries.

Note that removing additional classification fields of $\mathcal{K}^{-\{2,3\}}$ based on ranges can significantly reduce space complexity since the same $\mathcal{K}^{-\{2,3\}}$ is sufficient to represent $\mathcal{K}$ in Example 2. Only one false-positive check of a matched rule $R_{i}^{-\{2,3\}}$ is required. To further improve performance, the removed fields of $R_{i}$ can be checked in parallel.

Theorem 2 (FIELdS Reduction). Let $\mathcal{K}^{-m}$ be a classifier that results from an order-independent classifier $\mathcal{K}$ by removing $m$ fields, i.e., each $R=\left(I_{1}, \ldots, I_{k}\right)$ is replaced with $R^{-m}=\left(I_{1}, \ldots, I_{k-m}\right)$. If $\mathcal{K}^{-m}$ is order-independent then $\mathcal{K}^{-m}$ with a false-positive check of a single matched rule is a semantically equivalent representation of $\mathcal{K}$.

Proof. If a header $H$ does not match $\mathcal{K}^{-m}, H$ cannot match $\mathcal{K}$ (we only remove new constraints). If a header $H$ matches $R_{j}^{-m}$, $H$ can match only $R_{j}$ or $H$ does not match $\mathcal{K}$ (if a header matched both $R_{i}^{-m}$ and some $R_{j}, i \neq j$, it would contradict the orderindependence of $\mathcal{K}^{-m}$ ).

By Theorem 2, detecting a false-positive match of a rule $R_{i}^{-m} \in$ $\mathcal{K}^{-m}$ requires only to check $m$ additional fields of $R_{i}$. If a false positive is found the catch-all rule is used. At most one rule can match in $\mathcal{K}^{-m}$ classifiers, which hugely impacts the complexity and feasibility of implementation.

There are significantly different complexities involved in adding and removing fields with the suggested approach. In the first case it suffices to maximize the size of the order-independent part on the same set of fields. In the second case, there is an additional tradeoff between the size of the order-independent part and the subset of fields necessary to retain order-independence. In the following section we suggest efficient representations of order-independent parts. The effect of Theorem 2 will be demonstrated in Section 8 .

\section{EFFICIENT REPRESENTATIONS OF CLASSIFIERS}

Since the tradeoff between lookup time and memory space for multi-field classification heavily depends on the number of fields $k$ participating in classification, it is reasonable to explore ways to reduce $k$. For software-based solutions, any additional field that is represented by a prefix or range incurs additional complexity on the lookup time or memory space [26]. If the reduced classifier $\mathcal{K}^{-m}$ contains at most two fields, we can efficiently implement lookup in time logarithmic in $N$ with linear memory [36]. For TCAM-based solutions, if a classifier field is represented by a range, removing it reduces the required TCAM space proportionally to the number of prefixes used to express the range. In this section we present optimization problems as a step to achieve this goal. Moreover, removing fields can create a shorter classification format. Common TCAM formats include 72, 144, or 288 bits, so reducing the representation size across one of those barriers halves the required TCAM space.

\subsection{Semantically Equivalent Representations of All Rules}

We begin by optimizing order-independent classifiers based on Theorem 2 (see Example 2).

\section{Problem 1 (Fields Subset Minimization (FSM)).}

Find a maximal subset of fields $M$ of an order-independent classifier $\mathcal{K}$ such that $\mathcal{K}^{-M}$ is order-independent; if there are several such subsets, choose $M$ with maximal total width (to minimize lookup word width).

FSM can only be applied to order-independent classifiers, and it does not always reduce the number of classification fields for orderindependent classifiers. It is enough that every field of a single rule is required to keep order-independence of a classifier. One way to address these shortcomings is a multi-group representation. A subset of classification fields may suffice for order-independence of a subset of rules of a classifier. Assume that the rules of a classifier $\mathcal{K}$ can be assigned to $\beta$ groups such that: (1) each rule belongs to a single group; (2) the rules of each group are order-independent on a subset of $k$ fields of $\mathcal{K}$ (except the "catch-all" rule); (3) different groups can reuse the same fields to keep order-independence. By Theorem 2, a lookup to a group returns a single matched rule to be checked for false-positives on all remaining fields; the matched non-false-positive rule with the highest priority (from exactly $\beta$ matched rules) is returned. We call this the multi-group representation of $\mathcal{K}$. Multi-group representations exist for any orderdependent classifier, but each group should be order-independent. The following theorem immediately follows by construction.

THEOREM 3. The multi-group representation of a classifier is semantically equivalent to the original classifier.

Lookups to groups can be issued in parallel. If each group in a multi-group representation can be order-independent on at most two fields, we have a semantically equivalent representation of a classifier in linear memory and with worst-case guaranteed logarithmic lookup time. Example 3 shows how the rules of a classifier $\mathcal{K}$ can be split into groups.

EXAMPLE 3. Consider $\mathcal{K}=\left\{R_{1}, R_{2}, R_{3}, R_{4}, R_{5}\right\}$ with three fields of four bits each and rules

$$
\begin{aligned}
& R_{1}=([5,10],[4,7],[4,5]), \\
& R_{2}=([1,4],[4,7],[4,5]), \\
& R_{3}=([1,9],[1,3],[4,6]), \\
& R_{4}=([1,9],[4,7],[1,3]), \\
& R_{5}=([1,9],[4,7],[5,6]) .
\end{aligned}
$$

While the set of all rules $\left\{R_{1}, R_{2}, R_{3}, R_{4}, R_{5}\right\}$ is not order independent (for instance, $R_{1} \cap R_{5} \neq \emptyset$ ), the rules can be divided into two independent subsets, $\left\{R_{1}, R_{2}, R_{3}\right\}$ and $\left\{R_{4}, R_{5}\right\}$. 


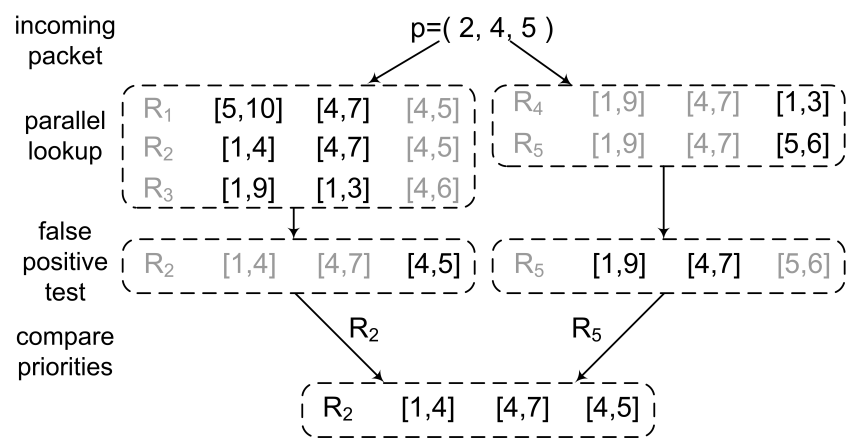

Figure 4: A visualization of the lookup procedure with a multigroup representation of the rules in Example 3. Fields to be checked on a certain step are in black; those that are irrelevant for this step, in grey.

The first two fields are necessary to keep the first group orderindependent and the last field is enough to keep the second group order-independent.

Figure 4 shows a visualization of the lookup procedure after the grouping has been accomplished: for an incoming packet $p=(2,4,5)$, we issue parallel lookups for the two groups; these lookups return $R_{2}$ and $R_{5}$ and look only at fields $\{1,2\}$ and $\{3\}$ respectively. Then we check if it was a false positive, matching $p$ against the rest of the fields for the returned rules, and then select the match by rule priority. At most one rule will make it to the false positive test for each group. In case when no rule matches or the final test finds that the result was a false positive, the catch-all rule is sent to the priority comparison, so each group sends exactly one rule to the priority comparison for any packet.

In some order-independent classifiers removing any of the fields make it be order-dependent and accordingly the FSM cannot reduce the number of fields. This leads us to the following optimization problem that can be applied also to order-dependent classifiers.

Problem 2 ( $l$-Groups of RUles ( $l$-MGR)). Given $a$ classifier $\mathcal{K}$, find an assignment of $\mathcal{K}$ 's rules to a minimal number of disjoint groups such that different groups of rules can be based on the same or different subset of at most $l \leq k$ fields, and each group is order-independent on these fields.

\subsection{Representation of a Subset of Rules}

Problem 2 shows how to deal with order-dependent classifiers. However, the number of lookups that can be issued in parallel is a system parameter, and the minimal number of groups found by solving Problem 2 may be unacceptable. In this situation, we can decompose an order-dependent classifier $\mathcal{K}$ into order-independent part $\mathcal{I}$ and order-dependent part $\mathcal{D}$. We can perform parallel lookups on both parts and return the best matched rule. TCAM or another general implementation can be used to store $\mathcal{D}$. Since in practice a subset of order-dependent rules on $k$ fields should be significantly smaller than $N$, significant TCAM space can be saved. The problem is finding which rules to put in $I$. One possibility is to find a maximal order-independent subset of rules on all $k$ fields and run Problems 1 or 2 on $\mathcal{I}$. Finding maximal order-independent set on $k$ fields is interesting when we extend already existing classifiers with new fields (Theorem 1). Another direction is finding an order-independent part that already has the desired properties.

Problem 3 (MaXimum Rules Coverage ( $l$-MRC)). Given a classifier $\mathcal{K}$ and a positive number $l \leq k$, find a maximal subset $\mathcal{I} \subseteq \mathcal{K}$ which is order-independent on at most l fields.

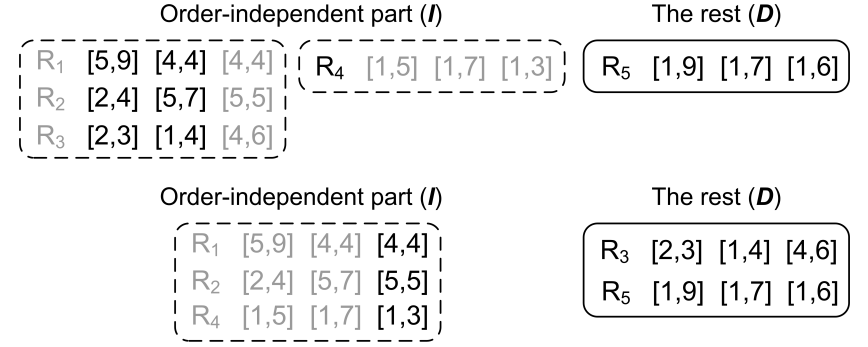

Figure 5: Example 5. Top: a multi-group representation of $\mathcal{I}=$ $\left\{R_{1}, R_{2}, R_{3}, R_{4}\right\}$ and $\mathcal{D}=\left\{R_{5}\right\}$. Bottom: a more compact representation of $\mathcal{I}=\left\{R_{1}, R_{2}, R_{4}\right\}$ and $\mathcal{D}=\left\{R_{3}, R_{5}\right\}$. Fields to be checked in a group lookup are in black; those that are irrelevant for this step, in grey.

EXAmPle 4. Consider a classifier $\mathcal{K}=\left\{R_{1}, R_{2}, R_{3}\right\}$ with three fields and the first three rules from Example 3 $R_{1}=$ ([5, 10], [4, 7], [4, 5]), $R_{2}=([1,4],[4,7],[4,5]), R_{3}=$ $([1,9],[1,3],[4,6])$. If $l=2$, we can have a maximal subset $\mathcal{K}^{\prime}=\mathcal{K}$ of three rules, as the first two fields guarantee that the set $\mathcal{K}$ of these three rules is order-independent. Likewise, if $l=1$ and only one field can be selected to distinguish between the rules, we can have order-independent sets of two rules. By considering the first field, we can have the subset $\mathcal{K}^{\prime}=\left(R_{1}, R_{2}\right)$. Using only the second field we can have other possible order-independent subsets such as $\mathcal{K}^{\prime}=\left(R_{1}, R_{3}\right)$ or $\mathcal{K}^{\prime}=\left(R_{2}, R_{3}\right)$. We can also see that the three rules together are not order-independent based on any single field and accordingly the three mentioned possible subsets are all maximal subsets.

The generalized version of $l$-MRC problem reuses the advantages of the decomposition into $I$ and $D$ and multi-groups representation.

Problem $4 \quad((\beta, l)$-MRC). Given a classifier $\mathcal{K}$ and two positive numbers $l \leq k$ and $\beta$, find a maximal subset of rules $\mathcal{I} \subseteq \mathcal{K}$ that can be assigned to at most $\beta$ groups, where each group is order-independent on at most l fields.

In some cases, it may be feasible to send a few more rules to $\mathcal{D}$ and thus significantly reduce the number of groups; this usually happens in practice with more general rules at the bottom of a classifier.

EXAMPLE 5. Consider a classifier $\mathcal{K}$ with three fields of five bits each and five rules

$$
\begin{aligned}
& R_{1}=([5,9],[4,4],[4,4]), \\
& R_{2}=([2,4],[5,7],[5,5]), \\
& R_{3}=([2,3],[1,4],[4,6]), \\
& R_{4}=([1,5],[1,7],[1,3]), \\
& R_{5}=([1,9],[1,7],[1,6]) .
\end{aligned}
$$

The maximal order-independent subset on all three fields is $\mathcal{I}=$ $\left\{R_{1}, R_{2}, R_{3}, R_{4}\right\}$, and one possible multi-group representation of $\mathcal{I}$ consists of two groups: $\left\{R_{1}, R_{2}, R_{3}\right\}$ (based on the first two fields) and $\left\{R_{4}\right\}$. But if we set $\mathcal{D}=\left\{R_{3}, R_{5}\right\}$, the rest will form a single group which is order-independent on a single field, the third one. This example is illustrated on Figure 5.

\subsection{Efficient Cache Implementation}

The $(\beta, l)$-MRC problem maximizes the number of rules in $\mathcal{I}$ that can be assigned to at most $\beta$ groups that are order-independent 
on at most $l$ fields. Previously, we discussed a decomposition of $\mathcal{K}$ into $\mathcal{I}$ and $\mathcal{D}$, returning the best match between them. We wish to construct $\mathcal{I}$ in such a way that if a non-catch-all rule of $\mathcal{I}$ is matched, the lookup to $\mathcal{D}$ is no longer required. If such $\mathcal{I}$ exists, its content can be configured in cache. If $\mathcal{K}$ is order-independent, $\mathcal{I}$ that is returned by the $(\beta, l)$-MRC problem satisfies the "cache" property. In the order-dependent case we just need to guarantee that there exist no two rules $R_{1} \in \mathcal{I}$ and $R_{2} \in \mathcal{D}$ such that $R_{1}$ "intersects" with $R_{2}$ with priority $R_{2} \prec R_{1}$. As a result, we formulate the following variant of the $(\beta, l)$-MRC problem.

Problem $5 \quad((\beta, l)$-MRCC). Given a classifier $\mathcal{K}$ and two positive numbers $l \leq k$ and $\beta$, find a maximal subset of rules $\mathcal{I} \subseteq \mathcal{K}$ that can be assigned to at most $\beta$ groups, where each group is order-independent on at most l fields and no two rules $R_{1} \in \mathcal{I}$ and $R_{2} \in \mathcal{D}$ such that $R_{1}$ "intersects" with $R_{2}$ and $R_{2} \prec R_{1}$.

The built $\mathcal{I}$ by $(\beta, l)$-MRCC has not only a space-efficient representation but also implements a power-efficient solution that does not require a lookup to $\mathcal{D}$ (if $\mathcal{I}$ is matched) that is usually will be implemented in TCAMs that suffer from power-inefficiency.

\subsection{Resolution of Optimization}

In case when only a part of the fields are represented by ranges or ranges are remapped to ternary bit-strings the boundaries of real classification fields as SrcIP, DstIP, ToS, etc., are removed. A rule becomes a ternary bitstring where any number of bits can be grouped to virtual fields.

EXAMPLE 6. Consider a classifier $\mathcal{K}$ with two fields of four bits each and four rules:

$$
\begin{aligned}
& R_{1}=\left(\begin{array}{ll}
100 *, & 001 *
\end{array}\right), \\
& R_{2}=\left(\begin{array}{ll}
1010, & 0001
\end{array}\right), \\
& R_{3}=\left(\begin{array}{lll}
000, & \star \star \star \star
\end{array}\right), \\
& R_{4}=\left(\begin{array}{lll}
001 *, & \star \star \star \star
\end{array}\right) .
\end{aligned}
$$

In this case, the first field of four bits is enough to ensure orderindependence, so if we run FSM on two four-bit fields, we get a classifier

$$
\begin{array}{ll}
R_{1}^{-1}=(100 *), & R_{2}^{-1}=(1010) \\
R_{3}^{-1}=(000 *), & R_{4}^{-1}=(001 *) .
\end{array}
$$

However, note that only the first and third bits suffice to provide order-independence in this case. Hence, if we treat the same $\mathcal{K}$ as 8 one-bit fields, FSM yields a classifier of width two:

$$
R_{1}^{-6}=(10), R_{2}^{-6}=(11), R_{3}^{-6}=(00), R_{4}^{-6}=(01) .
$$

We have performed experiments on how the resulting reduced classifier width depends on the width of virtual fields. The results are summarized in Figure 6 that shows average widths of ClassBench and CISCO classifiers for different resolutions.

In light of Example 6, solutions to problems in this section are very promising even for the case of a single field represented by a range. This is actually the case of minimization of representation in forwarding tables. Since every prefix corresponds to a single range, finding a maximal order-independent set of ranges is equivalent to maximum independent sets in interval graphs, i.e. a graph in which the nodes are 1-dimensional intervals (e.g., time intervals) and there is an edge between two intervals iff they intersect. This problem can be solved exactly in $O(N \log N)$ time using the earliest-deadline-first (EDF) algorithm [17].

Using the order-independent property of a classifier can in some sense reduce its representation memory size beyond the theoretical entropy lower bounds presented in [27]. Of course, this relates to

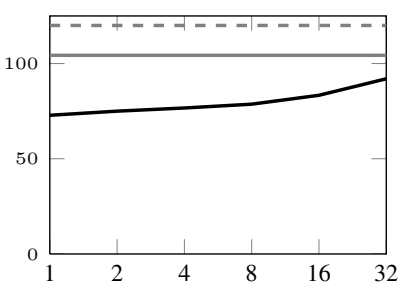

Virtual Field Width (in bits), ClassBench

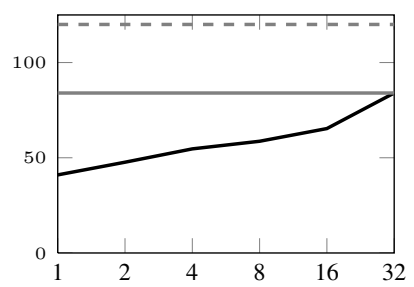

Virtual Field Width (in bits), CISCO
Figure 6: Classifier width (in bits) as a function of virtual field width for ClassBench and CISCO classifiers. Dashed grey line presents average original classifier width; grey solid line shows average classifier width after MinDNF reduction (see Section 5); black line illustrates classifier width after FSM optimization.

the space required in the first step of the classification and does not include the additional memory required to examine the case of a false-positive match.

Consider for instance, a classifier with four rules $R_{1}, R_{2}, R_{3}, R_{4}$ defined on a single field of $W=8$ bits such that $R_{1}=$ $[148,148]=(10010100), R_{2}=[83,83]=(01010011), R_{3}=$ $[165,165]=(10100101), R_{4}=[102,102]=(01100110)$. The rules are associated with four distinct actions $A_{1}, A_{2}, A_{3}$ and $A_{4}$. We can see that two bits are enough in order to distinguish between the rules. These can be for instance the third and the seventh bits which have the values of $00,01,10$ and 11 in the four rules $R_{1}, R_{2}, R_{3}, R_{4}$, respectively. Accordingly, the representation of this subset of the bits of the rules together with the encoding of the action of each rule (in two additional bits) has a total space of $4 \cdot(2+2)=16$ bits.

For this classifier, the binary tree representation $T$ includes 4 leaves and a total of $27<(32=4 \cdot W)$ nodes since some nodes for the first bits in the rules are shared among more than one rule. The suggested $X B W-l$ transform [27] for the tree, denoted $\mathrm{xbwl}(T)$, consists of three strings $\left(S_{\text {last }}, S_{I}, S_{\alpha}\right)$, where the bit length of the binary strings $S_{\text {last }}, S_{I}$ that encodes tree structure equals the number of nodes. Likewise, the length of string $S_{\alpha}$ that encodes the actions equals the number of leaves times the size of each action in bits. This results in a space requirement of $27+27+4 \cdot 2=62$ bits, almost four times the space required in the suggested scheme. Furthermore, the DAG representation [27], a more complicated encoding scheme for the tree $T$, cannot reduce the space required since in this example actions of the rules are disjoint and the tree does not have any isomorphic subtrees. In future work, we plan to compare the size of forwarding table representations computed by heuristics proposed in [27] with the results of semantically equivalent representations considered in this section. We believe that results for representations of IPv6 forwarding tables should be even better since in wider classifiers there is a better chance to find more order-independent rules on fewer bits.

\section{REPRESENTING CLASSIFIERS BOOLEAN EXPRESSIONS}

In this section, we discuss a special case when each field is represented by a prefix, and thus fields can be represented by a string of individual bits. This special case lets us represent classifiers as Boolean expressions and apply well known Boolean optimization techniques to reduce the width and number of rules.

We can represent rules and sets of rules as discrete-valued functions. For the purposes of this exposition, we concatenate all fields 
and treat rules as a ternary bit-string in the Value-Mask-Action format (since fields have a fixed width, we do not lose information with this transformation). In this part we consider the case of order-independent classifiers; we decide whether an action should be applied to an incoming packet, i.e., compute a Boolean function.

A rule $s=s_{1} s_{2} \ldots s_{k}, s_{j} \in\{0,1, *\}$, can be expressed as a conjunction $f_{s}\left(x_{1}, \ldots, x_{k}\right)=\bigwedge_{s_{i}=1} x_{i} \wedge \bigwedge_{s_{i}=0} \bar{x}_{i}$. Further, an order-independent set of rules $\mathcal{R}=\left\{R_{1}, \ldots, R_{N}\right\}$ with rules $s_{1}, \ldots, s_{k}$ can be written as an unordered disjunction of individual rules, i.e., as a formula of depth 2 in disjunctive normal form (DNF, disjunction of conjunctions).

EXAMPLE 7. The following set of rules with width 5

$\begin{array}{llllll}(0 & 1 & * & * & *) & \rightarrow 1 \\ (* & 1 & 0 & * & *) & \rightarrow 1 \\ (* & 1 & 1 & * & 0) & \rightarrow 1 \\ (* & 1 & 1 & * & 1) & \rightarrow 1\end{array}$

is order-independent and equivalent to $f\left(x_{1}, x_{2}, x_{3}, x_{4}, x_{5}\right)=$ $\left(\bar{x}_{1} \wedge x_{2}\right) \vee\left(x_{2} \wedge \bar{x}_{3}\right) \vee\left(x_{2} \wedge x_{3} \wedge \bar{x}_{5}\right) \vee\left(x_{2} \wedge x_{3} \wedge x_{5}\right)$

Thus, minimizing order-independent TCAMs is equivalent to the following problem.

PRoBlem 6 (MINDNF). For a given Boolean function $f$, find a minimal size DNF representation for $f$.

DNF minimization may result in both reducing the number of rules (merging clauses in the DNF) and reducing the width of lookup necessary for classification (removing extra variables in the clauses).

EXAMPLE 8. Let us minimize the set of rules from Example 7. We apply the well-known resolution heuristic to clauses in the DNF representation of $f$ :

$$
\begin{aligned}
\left(x_{1} \wedge \bar{x}_{2} \wedge \bar{x}_{5}\right) \vee\left(x_{1} \wedge \bar{x}_{2} \wedge x_{5}\right) & =\left(x_{1} \wedge \bar{x}_{2}\right) \\
\left(x_{1} \wedge \bar{x}_{2}\right) \vee\left(x_{1} \wedge \bar{x}_{2}\right) & =x_{2} .
\end{aligned}
$$

At this point, we have $f\left(x_{1}, x_{2}, x_{3}, x_{4}, x_{5}\right)=\left(\bar{x}_{1} \wedge x_{2}\right) \vee$ $x_{2}$, and we can subsume the first clause into the second: $f\left(x_{1}, x_{2}, x_{3}, x_{4}, x_{5}\right)=x_{2}$. After minimizing the corresponding DNF expression, we can express the result as a set of rules, a single rule in this case:

$$
\left(\begin{array}{lllll}
* & * & * & *
\end{array}\right) \rightarrow 1
$$

Thus, in this example we have reduced the number of rules from four to one and have reduced the number of bits participating in the lookup from four (to apply the rules in Example 7 as stated, we would have to query all bits except bit 4 ) to one (bit 2).

The MinDNF problem has been extensively studied in complexity theory. Its decision version (given a formula $\varphi$ in DNF and a number $k$, find whether there exists an equivalent DNF with size less than $k$ ) is unlikely to be in NP. It is obviously in $\mathrm{NP}^{\mathrm{NP}}$ : to solve this problem, one needs to guess a small DNF and verify with the NP oracle that solves the satisfiability problem that these two formulas are indeed equivalent. In fact, MinDNF has been shown to be $\mathrm{NP}^{\mathrm{NP}}$-complete ( $\Sigma_{2}^{P}$-complete), and some inapproximability results have also been proven $[16,37]$. In practice, classical heuristics for MinDNF were based on Karnaugh maps and the Quine-McCluskey algorithm.

A different view of the problem appeared for the case of functions given by truth tables [1]; this means that the input size is exponential in the number of variables, and an algorithm is thus also allowed to use exponential memory. In this case, one can represent MinDNF as a special case of the SetCover problem which leads to using the Greedy SetCover algorithm to find minimal DNFs (see Algorithm 3). This algorithm has been shown to be $O(n)$ approximate, where $n$ is the number of variables in the formula (for functions given by truth tables, $O(\log T)$-approximate, where $T$ is the truth table size), and a matching lower bound has also been provided [1]. However, this approach is impractical for classifier optimization since practical classifiers look up several hundred bits, and it would be infeasible to construct the truth table explicitly.

At the first glance MinDNF is a more general problem than FSM since it can reduce both classification width and the number of rules. However, due to an additional check for a false-positive match FSM can significantly reduce classification width beyond optimal results of MinDNF.

EXAMPLE 9. Consider the classifier from Example 6:

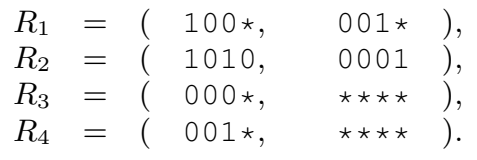

As we have discussed in Example 6, FSM can reduce the width of $\mathcal{K}$ from eight bits to four (if we treat fields as indivisible) or even two (if we are able to get down to the bit level). On the other hand, the only MinDNF heuristic applicable here is the resolution rule that can be applied to $R_{3}$ and $R_{4}$, getting

$$
\begin{aligned}
& R_{1}=\left(\begin{array}{ll}
100 *, & 001 *),
\end{array}\right. \\
& R_{2}=\left(\begin{array}{ll}
1010, & 0001
\end{array}\right) \text {, } \\
& R_{3}^{\prime}=(00 \star \star, \quad \star \star \star \star) \text {. }
\end{aligned}
$$

This classifier has width 8 ; if we discard bits with identical values (second bit in the first field), we get width 7 , but the width savings are still small compared to FSM.

Table 2 shows how MinDNF heuristics apply to our experimental set of classifiers; we have applied MinDNF to the orderindependent set from Table 1 . The number of binary rules is sometimes significantly larger than the number of original rules, and MinDNF does not have a significant effect on width, i.e., few bits become purely "don't care" bits even after MinDNF-style reductions. In Table 2, we show both "pure" width (cutting out bits that are purely "don't care") and further reduced width where we have cut out bits that have the same value, not necessarily "don't care". This is a Boolean counterpart of our reduction shown in Section 3 since we can use a single look up to check bits that always have the same value, and they do not change which rule matches an incoming packet. MinDNF does not significantly decrease width even with this reduction.

The example of FSM shows us that algorithms with usage of an additional constant number of checks for false-positive match can significantly reduce a required space of an optimal representation of Boolean expressions. We believe that developing new Boolean expression minimization techniques that are based on the results of Theorem 2 is a very promising direction.

\section{PROPRIETARY HEURISTICS}

The reduction in classifier size with a false-positive check opens new horizons that are not addressed by general Boolean expression minimization. In this section we study the complexity of the algorithms for the problems defined in Section 4. 


\begin{tabular}{|c|c|c|c|c|c|c|c|c|c|c|c|c|}
\hline & \multicolumn{5}{|c|}{ Original classifier } & \multicolumn{6}{|c|}{ MinDNF reduced } & \multirow{2}{*}{$\begin{array}{l}\text { Order-indep. } \\
\text { width, } \\
\text { bits }\end{array}$} \\
\hline & $\begin{array}{c}\text { Rules, } \\
\text { orig. }\end{array}$ & $\begin{array}{c}\text { Rules, } \\
\text { order-ind. }\end{array}$ & $\begin{array}{l}\text { Rules, } \\
\text { binary }\end{array}$ & $\begin{array}{l}\text { Rules, } \\
\text { SRGE }\end{array}$ & $\begin{array}{l}\text { Width, } \\
\text { bits }\end{array}$ & $\begin{array}{l}\text { Rules, } \\
\text { binary }\end{array}$ & $\begin{array}{l}\text { Width, } \\
\text { bits }\end{array}$ & $\begin{array}{l}\text { Red. wid., } \\
\text { bits }\end{array}$ & $\begin{array}{l}\text { Rules, } \\
\text { SRGE }\end{array}$ & $\begin{array}{c}\text { Width, } \\
\text { bits }\end{array}$ & $\begin{array}{l}\text { Red. wid., } \\
\text { bits }\end{array}$ & \\
\hline acl1 & 49870 & 49779 & 67511 & 65240 & 120 & 67505 & 90 & 90 & 65234 & 90 & 90 & 31 \\
\hline acl2 & 47276 & 44178 & 90772 & 90772 & 120 & 90233 & 104 & 104 & 90230 & 104 & 104 & 82 \\
\hline $\mathrm{acl} 3$ & 49859 & 47674 & 85252 & 83630 & 120 & 85226 & 106 & 106 & 83605 & 106 & 106 & 91 \\
\hline acl4 & 49556 & 46670 & 77837 & 76424 & 120 & 77755 & 106 & 106 & 76338 & 106 & 106 & 97 \\
\hline acl5 & 40362 & 38962 & 47514 & 47514 & 120 & 46261 & 96 & 94 & 46249 & 96 & 94 & 63 \\
\hline fw1 & 47778 & 43675 & 159525 & 159519 & 120 & 159461 & 112 & 112 & 159458 & 112 & 112 & 72 \\
\hline fw2 & 48885 & 48826 & 92646 & 92646 & 120 & 92316 & 88 & 88 & 92316 & 88 & 88 & 52 \\
\hline fw3 & 46038 & 41615 & 122495 & 122477 & 120 & 122259 & 112 & 112 & 122244 & 112 & 112 & 84 \\
\hline fw4 & 45340 & 42857 & 278887 & 277921 & 120 & 277799 & 104 & 104 & 276807 & 104 & 104 & 76 \\
\hline fw5 & 45723 & 39962 & 99574 & 99412 & 120 & 99421 & 112 & 112 & 99273 & 112 & 112 & 76 \\
\hline ipc1 & 49840 & 48294 & 66718 & 65734 & 120 & 66715 & 112 & 112 & 65731 & 112 & 112 & 50 \\
\hline ipc2 & 50000 & 50000 & 50000 & 50000 & 120 & 50000 & 112 & 112 & 50000 & 112 & 112 & 36 \\
\hline ciscol & 584 & 538 & 603 & 603 & 120 & 475 & 104 & 86 & 474 & 104 & 86 & 52 \\
\hline $\operatorname{cisco} 2$ & 269 & 249 & 565 & 565 & 120 & 564 & 104 & 84 & 564 & 104 & 84 & 21 \\
\hline $\operatorname{cisco} 3$ & 95 & 92 & 92 & 92 & 120 & 92 & 88 & 68 & 92 & 88 & 68 & 30 \\
\hline $\operatorname{cisco} 4$ & 364 & 329 & 629 & 629 & 120 & 629 & 104 & 84 & 629 & 104 & 84 & 38 \\
\hline $\operatorname{cisco} 5$ & 148 & 120 & 139 & 139 & 120 & 139 & 104 & 84 & 139 & 104 & 84 & 17 \\
\hline
\end{tabular}

Table 2: Experimental results of MinDNF reduction in the order-independent subsets of classifiers.

\subsection{Exact Algorithms}

We first consider the existence of algorithms to build exactly the proposed semantically equivalent representations of classifiers.

FSM is NP-complete by reduction from the SetCover problem. Since FSM is exponential on $k$, an exact algorithm is feasible only for a sufficiently small number of fields (e.g., the 5-tuple case).
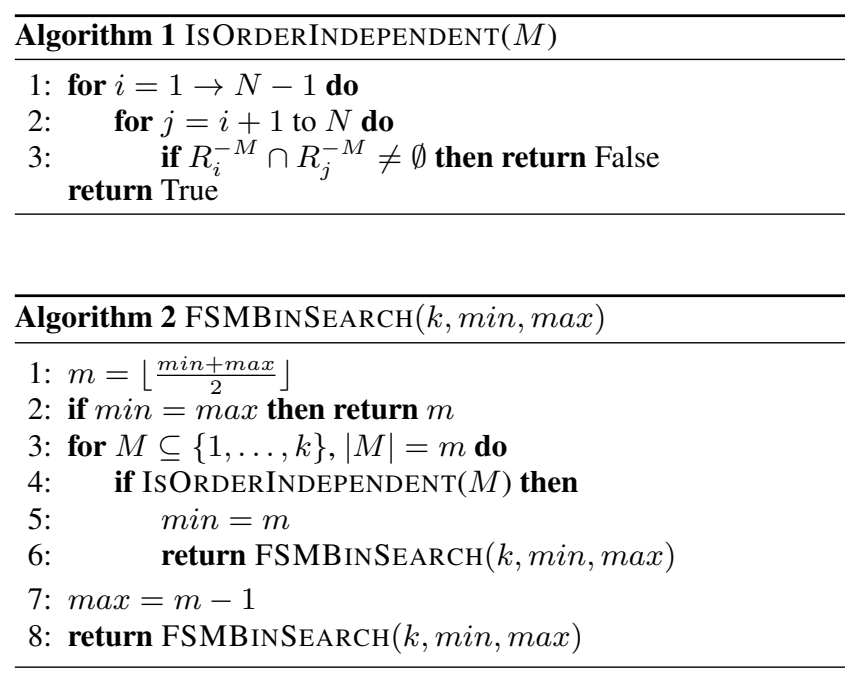

Algorithm 1 checks order-independence of a classifier with $N$ rules and a set $M$ of $m$ removed fields with complexity $O\left(N^{2}(k-\right.$ $m)$ ). To solve FSM, we can use a binary search. If the classifier is order-independent for some removed subset with $\frac{k}{2}$ fields, try to find a subset of size $\frac{3 k}{4}$, otherwise check order-independence for subsets of size $\frac{k}{4}$, and so on. We call this algorithm FSMBINSEARCH. At worst we need to run IsORDERINDEPENDENT once for each subset of size $\frac{k}{2}$, once for each subset of size $\frac{3 k}{4}$ and so on, $\left(\begin{array}{c}k \\ k / 2\end{array}\right)+\left(\begin{array}{c}k \\ k / 4\end{array}\right)+\ldots+\left(\begin{array}{c}k \\ 1\end{array}\right)<2^{k-1}$ times in total. Thus, we get the following theorem.

THEOREM 4. FSMBINSEARCH $(k, 0, k-1)$ finds a minimal subset of $k$ fields for which an original classifier is orderindependent in time $O\left(k 2^{k-1} N^{2}\right)$.

When the number of fields grows (e.g, by a resolution increase as in Section 4.4 or adding new fields), FSM requires approximation heuristics.
The 2-MRC problem is NP-complete already for the case when $k=2$ [4]. Since the $(\beta, l)$-MRC problem simply generalizes the 2-MRC problem when $\beta=1, k=2$, we can directly deduce based on the hardness of the 2-MRC problem that the $(\beta, l)$-MRC is NP-complete as well. As considered in Section 4.4 for $k=1$, only the 1-MRC problem has an exact solution by EDF algorithm in $O(N \log N)$ time [17]. Unfortunately, even the 2-MRC problem is exponential on $N$, so we do not consider exact algorithms similar to FSM when a number of fields is small.

\subsection{Approximate Solutions}

Algorithms for the FSM problem are exponential in the number of fields $k$; exact algorithms for the other considered problems are exponential even in the number of rules $N$. Therefore, as $k$ and $N$ grow, approximations are needed. In this section, we discuss efficient approximate algorithms that are crucial to achieve TCAM space savings.

\subsubsection{Representation of All Rules}

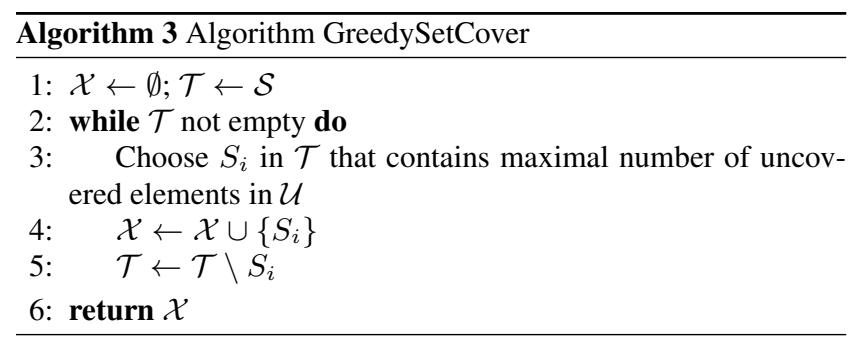

THEOREM 5. FSM is reducible to SetCover in $O\left(k \cdot N^{2}\right)$ time and has an approximation factor of $2 \cdot \ln (N)+1$.

Proof. Given an instance of the FSM problem, i.e., a set of $N$ rules $R_{1}, \ldots, R_{N}$, each with $k$ fields, we would like to find a subset of the fields that can distinguish between any pair of rules. A field distinguishes between two rules if they do not intersect in the field. We look at the (unordered) possible pairs of rules as the universe $U$ that we would like to cover, i.e., to make sure that each of these pairs of rules can be distinguished by one of the fields. We define $U=\{(i, j) \mid i<j, i, j \in[1, N]\}$. The size of $U$ is $|U|=\left(\begin{array}{c}N \\ 2\end{array}\right) \leq 1 / 2 \cdot N^{2}$. We also define $k$ sets $S_{1}, \ldots, S_{k}$ that are used to cover $U$ such that the set $S_{\ell}$ represents the $\ell^{\text {th }}$ field out of $k$. For $\ell \in[1, k]$, we define $S_{\ell}=\{(i, j) \mid i<j, i, j \in$ 
$\left.[1, N], R_{i}(\ell) \cap R_{j}(\ell)=\emptyset\right\} . S_{\ell}$ contains all pairs of rules that do not intersect in this field. To construct $S_{1}, \ldots, S_{k}$, one has to check all pairs out of $N$ rules. Therefore, this construction requires a time complexity of $O\left(k \cdot N^{2}\right)$ - polynomial in size of the input to the FSM problem. Given a solution to the set cover instance, i.e., a subset of $S_{1}, \ldots, S_{k}$, the corresponding set of fields are guaranteed to distinguish between any pair of rules and therefore comprise an admissible solution to the FSM problem.

Using Algorithm 3, in which each step selects an additional set with maximal number of uncovered elements, we can achieve an approximate solution to the FSM problem. In such a solution, the number of selected fields is within a constant factor of the optimal number of fields. The approximation ratio is $H(|U|)=H\left(\left(\begin{array}{c}N \\ 2\end{array}\right)\right)$, where $H$ is the harmonic number, such that the approximation ratio satisfies $H(|U|) \leq \ln (|U|)+1 \leq 2 \cdot \ln (N)+1$.

Next we consider an estimate for a lower bound on $\beta$ (number of groups) in multi-group representations and how that bound relies on $N$ and $k$ for order-independent classifiers.

THEOREM 6. 1. For each $N$ and $k \geq 2$, there exists an orderindependent classifier $\mathcal{K}$ of $N$ rules on $k$ fields such that it cannot be divided into less than $\sqrt{N}-O(1 / \sqrt{N})$ groups where each group is order-independent by a single field.

2. Moreover, if $k \geq 4$, there exists $\mathcal{K}$ such that it cannot be divided into less than $\sqrt{N}-O(1 / \sqrt{N})$ groups where each group is order-independent by two fields.

3. Moreover, if $N \geq 2^{k}$, there exists $\mathcal{K}$ such that it cannot be divided into less than $2^{k-l}$ groups where each group is orderindependent by some l fields.

PROOF. 1. For some natural $n$, consider a classifier on two fields with $n(n-1)$ rules; the rules span all pairs of intervals $([i, i],[j, j])$ for $1 \leq i \neq j \leq n$. Any group of rules that are independent with respect to any single field cannot contain more than $n$ rules (since there are only $n$ distinct intervals in each field), so there are at least $n-1$ groups.

2. To extend this counterexample to pairs of fields, consider a classifier on 4 fields that contains $n(n-1)(n-2)(n-3)$ rules that implement all quadruples of intervals $([i, i],[j, j],[k, k],[l, l])$, $1 \leq i \neq j \neq k \neq l \leq n$. Now any group of rules that are independent with respect to any pair of rules can contain at most $n(n-1)$ rules, so there are at least $(n-2)(n-3)$ groups in total.

3. Now the example is a classifier consisting of $2^{k}$ rules that implement all possible combinations of two disjoint intervals (say, $[1,1]$ and $[2,2])$ in $k$ rules. Now any group independent with respect to $l$ fields can contain at most $2^{l}$ rules (exhausting all possible combinations of $[1,1]$ and $[2,2]$ of length $l$ ), so there must be at least $2^{k-l}$ such groups.

In Section 6.2.2 we propose a simple heuristic to resolve Problem 2 and evaluate its performance in Section 8.

\subsubsection{Representation of a Maximal Number of Rules}

For the case of $k=2$, the 2-MRC problem can be approximated with factor $O(\log \log N)$ [4]. Consider the $l$-MRC problem in the case of $l=k$, i.e., finding a maximal subset of rules order-independent on all $k$ fields. In this special case, the problem reduces to the maximum independent set problem where each rule represents a node and an edge between two rules exists iff they intersect by some field.

In Theorem 5, we showed how the FSM problem reduces to SetCover; in order to find an independent set of rules based on a subset of the fields, we must make sure that each pair of rules does not intersect on at least one of the fields and defined the corresponding coverage problem. We considered the coverage of a field as the set of pairs of rules it can separate. Unfortunately, it is not straightforward to make use of the same idea in order to reduce the $l$-MRC problem to the MSC because a field that separates the maximal number of pairs of rules does not necessarily yield the maximal size of an independent set of rules.

For example, consider the following classifier $\mathcal{K}=$ $\left(R_{1}, R_{2}, R_{3}, R_{4}\right)$ with four rules of two fields: $R_{1}=$ $([0,1],[0,0]), R_{2}=([2,3],[1,1]), R_{3}=([0,1],[2,2]), R_{4}=$ $([2,3],[0,3])$. There are four pairs of rules that do not intersect in the first field. These are pairs $\left(R_{1}, R_{2}\right),\left(R_{1}, R_{4}\right),\left(R_{2}, R_{3}\right)$, $\left(R_{3}, R_{4}\right)$. Likewise, there are three pairs of rules with disjoint second field: $\left(R_{1}, R_{2}\right),\left(R_{1}, R_{3}\right)$ and $\left(R_{2}, R_{3}\right)$. For this classifier, in the 1-MRC problem we should select the second field to obtain a maximal independent subset of rules although it distinguishes between fewer pairs of rules than the first field. Based only on the first field, the maximal set of independent rules includes two rules ( $\left\{R_{1}, R_{2}\right\},\left\{R_{1}, R_{4}\right\},\left\{R_{2}, R_{3}\right\}$, or $\left.\left\{R_{3}, R_{4}\right\}\right)$. Adding the second field, we get an independent set of three rules $\left\{R_{1}, R_{2}, R_{3}\right\}$ because we can separate all pairs of rules in it, so the size of the maximal independent set is not determined by the number of disjoint pairs of rules.

Nevertheless, we can use an algorithm for the $l$-MSC problem as a heuristic to solve the $l$-MRC problem. We give the pairs of rules each field can separate as input to an algorithm that solves $l$-MSC.

Problem 7 (Maximum Set Coverage ( $l$-MSC)). Given a set of elements $\mathcal{U}=\{1,2, \ldots N\}$ (the universe), a set $\mathcal{S}$ of $k$ sets whose union equals $\mathcal{U}$, and a positive number $l \leq k$, select at most $l$ sets of $\mathcal{S}$ such that as many elements of $\mathcal{U}$ as possible are covered (the union of selected sets has maximal size).

A variant of Algorithm 3 that stops after $l$ subsets are added solves the $l$-MSC problem and has an approximation factor of $1-\frac{1}{e}+o(1)[10]$.

To solve $(\beta, l)$-MRC, we can run $l$-MRC; once any unassigned rule "intersects" with any rule assigned to the current group a new group is opened if the total number of groups does not exceed $\beta$. The algorithm for $l$-MGR is the same as for $(\beta, l)$-MRC but now we stop to create new groups once we covered all rules of the original classifier.

The proposed heuristic for the $l$-MRC problem and its variant for $(\beta, l)$-MRC have worst-case guaranteed lookup time $O(\log N)$ and a linear space requirement when $l=2$. In practice there is an excess of information that preserves order-independence on a relatively small subset of bits (see Section 8)

\section{CLASSIFIER CONFIGURATION}

This section proposes new standard representations of classifiers and discusses updates.

\subsection{Standardization of Policy Classifiers}

We group classifiers into two categories: those required for switching and those implementing service-level-agreement (SLA) policies. The former type can be changed frequently, while the latter is typically static. Dynamic updates for the latter type are significantly less important and we can consider offline computation for more efficient representations.

We suggest explicitly specifying certain classifier characteristics (that can be computed offline) as part of a classifier configuration. The following traits (or variations thereof) are important: (1) maximal size of the order-independent part; (2) minimal subset of fields that preserve order-independence; (3) minimal number of orderindependent groups based on at most two fields; (4) assignments of 
rules to a predefined number of groups based on at most two fields. With these the optimal implementation can be chosen based on the constraints of a configured network element.

\subsection{Dynamic Updates}

Suppose that classifier represents packet flows that can change frequently. It is straightforward to remove a rule from the orderindependent part $\mathcal{I}$; for the order-dependent part $\mathcal{D}$, it depends on the representation. Insertion is more complicated. If an inserted rule $R$ is order-dependent with the current $\mathcal{I}$, insert $R$ to $\mathcal{D}$. If $\mathcal{D}$ is full, recompute $\mathcal{I}$ and $\mathcal{D}$ for the new set. If $\mathcal{D}$ is still full, reject $R$. If $R$ is order-independent with $\mathcal{I}$ on $k$ fields and does not increase the subset of fields in FSM, or there is an assignment to one of the groups in MGR without increasing the number of orderindependent groups, add $R$ to $\mathcal{I}$. To avoid long delays, recomputation of $\mathcal{I}$ and $\mathcal{D}$ can start in the background. In this case there is a tradeoff between the current optimality of $\mathcal{I}$ representation and the delay before inserting the next rule.

We can increase the chance of successfully inserting a new rule in $\mathcal{I}$. Recall that for order-independent classifiers we must check all $k$ fields for a matched rule to check for false-positive matches on a subset of the fields. We can define an additional parameter $C$ : the maximal number of rules that can be checked for false-positives in a line rate. Suppose an inserted rule is order-independent with each rule of the current $\mathcal{I}$ on $k$ fields and increases the number of fields for FSM or requires more groups to keep the order-independence of all groups for MGR. We can still assign $R$ to $\mathcal{I}$ if the number of rules to be checked for false-positives is at most $C$. By doing so, we increase $|\mathcal{I}|$ by up to a factor of $C$.

It is also desirable to support modifications in existing rules. If a rule in $\mathcal{I}$ is only modified in fields not required for orderindependence, we can simply change it in additional memory. If the change is in one of the fields required for order-independence, we have to make sure this rule does not intersect other rules in $\mathcal{I}$ after the change. If so, we can simply update this rule in the representation of $\mathcal{I}$, and if it now intersects with some other rule, we can move this modified rule to $\mathcal{D}$. In an offline process, we can recalculate the selection of fields to distinguish among rules to match the recent new rules. As in the rule insertion case, modification of a rule in $\mathcal{D}$ depends on the representation of $\mathcal{D}$.

EXAMPLE 10. Consider a classifier $\mathcal{K}=\left(R_{1}, R_{2}, R_{3}\right)$ with three fields of four bits each and rules

$$
\begin{aligned}
& R_{1}=([1,3],[4,8],[1,5]), \\
& R_{2}=([7,7],[1,8],[4,5]), \\
& R_{3}=([4,5],[6,9],[4,6]) .
\end{aligned}
$$

The first field suffices for order-independence, so we have $\mathcal{K}^{-\{2,3\}}=(([1,3]),([7,7]),([4,5]))$ and $\mathcal{I}=\left\{R_{1}, R_{2}, R_{3}\right\}$. Let us try to insert $R_{4}=([2,4],[2,2],[3,3])$; it is order-independent with $\mathcal{I}$ but requires the second field for order-independence with $R_{1}$ and $R_{3}$. In this (somewhat unlucky) case we can still use only the first field for $\mathcal{I}$ and test $R_{4}$ additionally if either $R_{1}$ or $R_{3}$ is matched (this requires $C \geq 2$ ). Note that it is not necessary to match $R_{4}$ if $R_{2}$ is matched since it is order-independent with $R_{2}$ on the first field. This example is illustrated on Figure 7.

\section{SIMULATIONS}

To validate our scheme, we run simulations on 12 classifiers from Classbench [7] (generated with real parameters), each with $\approx 50 \mathrm{~K}$ rules on 6 fields, and on 5 real life classifiers provided by Cisco Systems. In Figure 1 and Table 1, we have already presented

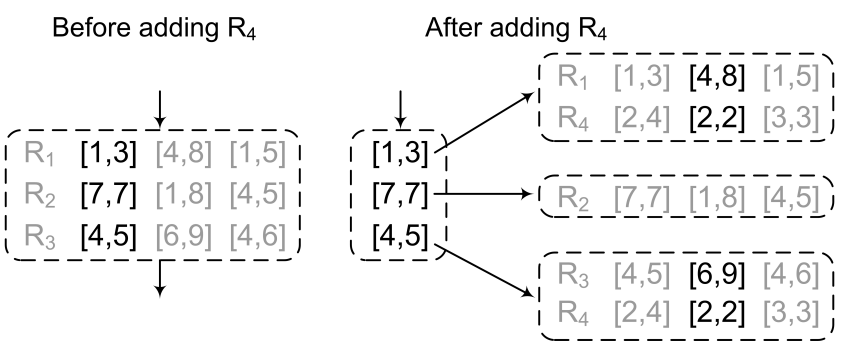

Figure 7: Example 10. Left: a multi-group representation of $\mathcal{I}=\left\{R_{1}, R_{2}, R_{3}\right\}$. Right: a representation of the two-step lookup procedure for $C \geq 2$ and recently inserted $R_{4}$. Fields to be checked in a group lookup are in black; those irrelevant in this step, in grey.

the TCAM space savings resulting from the FSM approach when adding new fields to these classifiers; we have shown that orderindependence greatly simplifies adding new fields to classifiers. In Table 2, we compared the proposed FSM approach with MinDNF heuristics, showing that FSM algorithms can have a much greater effect.

The next set of simulations deals with maximal orderindependent sets. We use the greedy algorithm for the $l$-MRC problem (see Section 6.2.2) to find a maximal order-independent set on all $k$ fields. Then we apply the FSM problem on the computed maximal order-independent set. To simulate Problem 2 ( $l$-MGR) with one- and two-field order-independent groups, we use the algorithm proposed in Section 6.2.2.

The results are summarized in Table 3. In most cases, the vast majority of rules are covered by very few groups of orderindependent rules. In some cases the maximal order-independent set constructed with a subset of fields $\mathcal{F}=\{0,1\}$ is larger than such a set constructed with all fields; this is due to a few rules with large intervals in the first two fields that have been taken up to the set because they are independent on some other field and prevented more rules to join the set later. For example, a rule of the form

0.0.0.0/0 0.0.0.0/0 $1234: 1234 \quad 0: 655350 \times 00 / 0 \times 00 \quad 0 \times 0000 / 0 \times 0000$ would block all further rules with source port 1234 if this port has not appeared earlier but would not be taken if only the IP addresses had been taken into account. Thus, interestingly, a greedy approach is not necessarily monotonic as the number of fields taken into account increases.

The effect explained in Example 5 is also seen in simulations. In many cases we see many very small groups (of size $\leq 2$ or $\leq 5$ ) created by general rules at the bottom of the lists; in practice it makes sense to send these rules to $\mathcal{D}$. Table 3 also shows that a maximal order-independent subset mostly takes care of the small groups, as very few such groups are left when running $l$-MGR on the resulting maximal order-independent set.

The majority of the proposed approximate algorithms are greedy without backtracking (the variants of GreedySetCover algorithm) with $|\mathcal{U}|=O\left(N^{2}\right)$, the running time of these algorithms is $O\left(k N^{2}\right)$, where $N$ is a number of rules and $k$ is a number of fields in a classifier. As a result we do not evaluate a computational time of the proposed algorithms.

\section{RELATED WORK}

Research towards efficient implementations of packet classifiers falls into two main categories: algorithmic solutions (usually software based) and TCAM-based solutions. Algorithmic solutions mainly rely on one of three techniques: decision trees, hashing, or coding-based compression. The works $[11,32,39]$ suggest how 


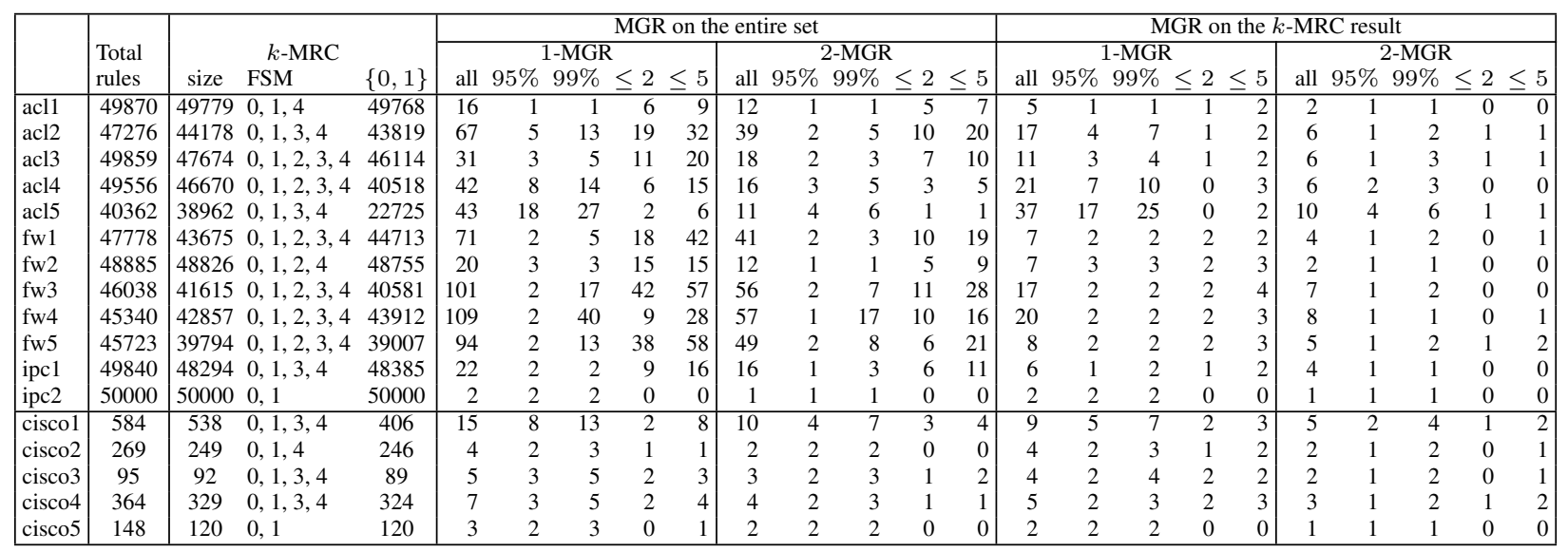

Table 3: Simulation results. Columns, left to right: total no. of rules $|\mathcal{K}| ;|\mathcal{I}|$, where $\mathcal{I}=\operatorname{MRC}(\mathcal{K},\{0, \ldots, 5\})$; minimal size subset of fields w.r.t. which $\mathcal{I}$ is order-independent; $\left|\mathcal{I}^{\prime}\right|$, where $\mathcal{I}^{\prime}=\operatorname{MRC}(\mathcal{K},\{0,1\})$ (i.e., order-independent w.r.t source IP and destination IP); for the one-field result $\{\mathcal{G}\}=M G R(\mathcal{K})$ : total number of order-independent groups $|\{\mathcal{G}\}|$, no. of groups covering $95 \%$ and $99 \%$ of the rules, no. of groups of size $\leq 2$ and of size $\leq 5$; similar statistics for the two-field MGR; similar statistics for one-field and two-field MGR run on $\mathcal{I}$.

to partition the multi-dimensional rule space. Possible matching rules are found by tracing a path in a decision tree. Techniques to balance the partition in each node exist, but rule replication often cannot be avoided. A related approach is described in [43]. There is an inherent tradeoff between space and time complexities in these approaches. Song and Turner's ABC algorithm for filter distribution offers higher throughput with lower memory overhead and the ability to tune the implementation for better time complexity or better space complexity [34]. The works [9,13] discuss hash-based solutions to match a packet to its possible matching rules. Efficient coding-based representations are shown in [27, 28,31]. Other works discuss efficient TCAM implementations. TCAMs do not natively support ranges, so one has to translate range fields into prefix representations. Bounds for the maximal number of TCAM entries required to encode a range have been proposed. A range of $W$ bits can be encoded in at most $2 W-2$ entries using only entries with a positive action ("accept") [36]. This bound improves to $2 W-4$ using gray coding [3]. Both schemes encode a multi-field range with a number of entries that is exponential in the number of fields $k$ with upper bounds of $(2 W-2)^{k}$ and $(2 W-4)^{k}$, respectively. When including negative action entries ("deny"), the upper bound is improved to $W$ entries [29]. The works [29,30] suggested schemes to encode a $k$-field range with complexity linear in $k$. Unfortunately, these schemes apply only to the encoding of a single rule; the encoding of a classifier with more rules requires changing the conventional TCAM architecture.

Several works have considered reducing the number of TCAM entries in the encoding of a given classifier by relying on heuristics $[5,23]$. These techniques include encoding the most common ranges with additional bits [6,21], applying block permutations [42], designing tree-based architectures [38], and redundancy removal [20]. While many of these heuristics could be improved with randomization [19], they usually limit the number of fields in a rule or perform badly as the number of fields increases. Rule disjointness is not a new topic; efficient schemes for classification and update supporting that rely on this property have been proposed in $[2,35,41]$.

Multi-group representations to reduce power consumption have appeared at $[22,33]$, while the space-time tradeoff for representation of hierarchical classifiers has been considered in [15]. The relation between packet classifiers and optimization of Boolean ex- pressions was recently studied in [18]. We also note that reusing order-independence can significantly simplify splitting of a classifier over several network elements $[12,14]$.

\section{CONCLUSION}

Packet classification depends on the number of entries $N$ in a classifier and the classification width $W$ of its rules. In fact, for a given number of entries width is the determining factor for lookup complexity. Once we pass to multi-field classification, width, and thus matching complexity, tend to grow significantly. This is especially the case for expressive classification with ranges. In practice, the rules contain excessive information to distinguish between $N$ entries. Various techniques have been proposed to determine similar patterns across table entries and represent and evaluate them efficiently (see, e.g., [27]).

In this work, we take a different route, identifying new properties of classifiers that let us ignore superfluous information in classification lookup. Our proposed concept of order-independence simplifies classifier matching by splitting the problem into two subproblems: (a) the rule selection problem which is based only on parts of rules (not their entire width) and (b) the matching of the selected rule which is based only on the remainder of the fields not considered in (a). As we have demonstrated, the proposed classifier representations can significantly outperform dedicated space minimization approaches. While the proposed techniques are motivated by packet classification problems and demonstrated thereon, we believe that our simple yet effective concepts can be reused for significant improvements also in neighboring areas (e.g., matching in databases or data mining) and can also open new lines of research in other areas (e.g., Boolean minimization).

\section{ACKNOWLEDGMENTS}

We thank the anonymous reviewers and our shepherd Ali Ghodsi for their insightful comments. The work of Patrick Eugster and Kirill Kogan was partially supported by Cisco Systems under grant "A Fog Computing Architecture". The work of Sergey Nikolenko was partially supported by the President Grant for Leading Scientific Schools NSh-3856.2014.1. 


\section{REFERENCES}

[1] E. Allender, L. Hellerstein, P. McCabe, T. Pitassi, and M. E. Saks. Minimizing DNF formulas and $A C_{d}^{0}$ circuits given a truth table. In IEEE Conference on Computational Complexity, 2006.

[2] A. Bremler-Barr, D. Hay, and D. Hendler. Layered interval codes for TCAM-based classification. In IEEE Infocom, 2009.

[3] A. Bremler-Barr and D. Hendler. Space-efficient TCAM-based classification using Gray coding. IEEE Trans. Computers, 61(1):18-30, 2012.

[4] P. Chalermsook and J. Chuzhoy. Maximum independent set of rectangles. In ACM-SIAM SODA, 2009.

[5] Y.K. Chang, C.I. Lee, and C.C. Su. Multi-field range encoding for packet classification in TCAM. In IEEE Infocom Mini-Conference, 2011.

[6] H. Che, Z. Wang, K. Zheng, and B. Liu. DRES: Dynamic range encoding scheme for TCAM coprocessors. IEEE Trans. Computers, 57(7):902-915, 2008.

[7] ClassBench: A packet classification benchmark. http://www.arl.wustl.edu/classbench/.

[8] Configuring IP ACLs. http://www.cisco.com/en/US/docs/switches/ datacenter/sw/4_1/nxos/security/configuration/guide/sec_ipacls.pdf.

[9] S. Dharmapurikar, H. Song, J. S. Turner, and J. W. Lockwood. Fast packet classification using bloom filters. In ACM/IEEE ANCS, 2006.

[10] U. Feige. A threshold of $\ln n$ for approximating set cover. $J$. ACM, 45(4):634-652, 1998.

[11] P. Gupta and N. McKeown. Classifying packets with hierarchical intelligent cuttings. IEEE Micro, 20(1):34-41, 2000.

[12] N. Kang, Z. Liu, J. Rexford, and D. Walker. Optimizing the "one big switch" abstraction in software-defined networks. In ACM CoNEXT, 2013.

[13] Y. Kanizo, D. Hay, and I. Keslassy. Optimal fast hashing. In IEEE Infocom, 2009.

[14] Y. Kanizo, D. Hay, and I. Keslassy. Palette: Distributing tables in software-defined networks. In IEEE INFOCOM, 2013.

[15] A. Kesselman, K. Kogan, S. Nemzer, and M. Segal. Space and speed tradeoffs in TCAM hierarchical packet classification. J. Comput. Syst. Sci., 79(1):111-121, 2013.

[16] S. Khot and R. Saket. Hardness of minimizing and learning DNF expressions. In IEEE FOCS, 2008.

[17] J. M. Kleinberg and É. Tardos. Algorithm design. Addison-Wesley, 2006.

[18] K. Kogan, S. I. Nikolenko, W. Culhane, P. Eugster, and E. Ruan. Towards efficient implementation of packet classifiers in sdn/openflow. In ACM HotSDN, 2013.

[19] N. Lesh and M. Mitzenmacher. Bubblesearch: A simple heuristic for improving priority-based greedy algorithms. Inf. Process. Lett., 97(4):161-169, 2006.

[20] A. X. Liu, C. R. Meiners, and Y. Zhou. All-match based complete redundancy removal for packet classifiers in TCAMs. In IEEE Infocom, 2008.

[21] H. Liu. Efficient mapping of range classifier into Ternary-CAM. In IEEE Hot Interconnects, 2002.
[22] Y. Ma and S. Banerjee. A smart pre-classifier to reduce power consumption of tcams for multi-dimensional packet classification. In ACM SIGCOMM, 2012.

[23] C. R. Meiners, A. X. Liu, and E. Torng. Bit weaving: A non-prefix approach to compressing packet classifiers in TCAMs. IEEE/ACM Trans. Netw., 20(2):488-500, 2012.

[24] Netlogic Microsystems. Content addressable memory. http://www.netlogicmicro.com.

[25] OpenFlow 1.3 specification, 2012. http://www.openflow.org/ wk/index.php/OpenFlow_1_3_proposal.

[26] M. H. Overmars and A. Frank van der Stappen. Range searching and point location among fat objects. $J$. Algorithms, 21(3):629-656, 1996.

[27] G. Rétvári, J. Tapolcai, A. Korösi, A. Majdán, and Z. Heszberger. Compressing ip forwarding tables: towards entropy bounds and beyond. In ACM SIGCOMM, 2013.

[28] O. Rottenstreich, A. Berman, Y. Cassuto, and I. Keslassy. Compression for fixed-width memories. In IEEE ISIT, 2013.

[29] O. Rottenstreich, R. Cohen, D. Raz, and I. Keslassy. Exact worst-case TCAM rule expansion. IEEE Trans. Computers, 62(6):1127-1140, 2013.

[30] O. Rottenstreich, I. Keslassy, A. Hassidim, H. Kaplan, and E. Porat. On finding an optimal TCAM encoding scheme for packet classification. In IEEE Infocom, 2013.

[31] O. Rottenstreich, M. Radan, Y. Cassuto, I. Keslassy, C. Arad, T. Mizrahi, Y. Revah, and A. Hassidim. Compressing forwarding tables for datacenter scalability. IEEE Journal on Selected Areas in Communications, 32(1):138-151, 2014.

[32] S. Singh, F. Baboescu, G. Varghese, and J. Wang. Packet classification using multidimensional cutting. In $A C M$ SIGCOMM, 2003.

[33] M. Somasundaram. Memory and power efficient mechanism for fast table lookup.

[34] H. Song and J. S. Turner. ABC: Adaptive binary cuttings for multidimensional packet classification. IEEE/ACM Trans. Netw., 21(1):98-109, 2013.

[35] V. Srinivasan, S. Suri, and G. Varghese. Packet classification using tuple space search. In ACM SIGCOMM, 1999.

[36] V. Srinivasan, G. Varghese, S. Suri, and M. Waldvogel. Fast and scalable layer four switching. In ACM SIGCOMM, 1998.

[37] C. Umans. The minimum equivalent DNF problem and shortest implicants. J. Comput. Syst. Sci., 63(4):597-611, 2001.

[38] B. Vamanan and T. N. Vijaykumar. TreeCAM: decoupling updates and lookups in packet classification. In $A C M$ CoNEXT, 2011.

[39] B. Vamanan, G. Voskuilen, and T. N. Vijaykumar. EffiCuts: optimizing packet classification for memory and throughput. In ACM SIGCOMM, 2010.

[40] G. Varghese. Network Algorithmics: An Interdisciplinary Approach to Designing Fast Networked Devices. Morgan Kaufmann, 2005.

[41] Z. Wang, H. Che, M. Kumar, and S. K. Das. CoPTUA: Consistent policy table update algorithm for TCAM without locking. IEEE Trans. Computers, 53(12):1602-1614, 2004.

[42] R. Wei, Y. Xu, and H. J. Chao. Block permutations in Boolean space to minimize TCAM for packet classification. In IEEE Infocom Mini-Conference, 2012.

[43] X. Zhao, Y. Liu, L. Wang, and B. Zhang. On the aggregatability of router forwarding tables. In IEEE INFOCOM, 2010. 